\title{
La ville anonyme face à la mer : du mirage au métissage
}

\author{
The Anonymous City Facing the Sea: From a Mirage to Miscegenation
}

\begin{abstract}
RÉSUMÉ
Notre présent article tourne autour de la question de l'analyse géocritique des espaces de la ville anonyme et de la mer chez les deux auteurs algériens : Mohamed Dib à travers son roman Cours sur la rive sauvage et Yasmina Khadra dans L'Olympe des infortunes. Ces deux écrivains mettent en lumière la dynamique binaire de la ville anonyme et de la mer. La fusion de ces deux espaces crée des mirages révélateurs du métissage de leurs écritures et de leur société. Cette hybridité textuelle dévoile une critique néfaste de la métropole et de l'ère de la mondialisation.

Mots-clés : villes anonymes, mer, mirage, métissage, fusion
\end{abstract}

\begin{abstract}
Our current article talks about the geocritical analysis of the spaces of the anonymous city and the sea argued by the two Algerian authors: Mohamed Dib through his novel Cours sur la rive sauvage and Yasmina Khadra in L'Olympe des infortunes. These two writers highlight the binary dynamics of the anonymous city and the sea. The fusion of these two spaces creates mirages revealing the intermingling of their writings and their society. This textual hybridization reveals a harmful criticism of the metropolis and the era of globalization.

Keywords: anonymous cities, sea, mirage, miscegenation, fusion
\end{abstract}

\section{Introduction}

La cité moderne étale son emprise sur la littérature. Le roman algérien de langue française porte en lui les traces indélébiles de la toute-puissance de la ville. Elle s'érige en maîtresse absolue, omniprésente et en perpétuel changement.

Mais la ville ne peut s'épanouir qu'une fois mise en parallèle avec la mer. Cette dernière, en mouvement continu, permet à la ville de se refléter mais en déformant ses courbes, elle l'aide à changer d'apparence. Elle crée des illusions diverses et laisse le mirage prendre forme.

Les villes chimériques s'échafaudent au sein de la diégèse. Elles naissent et meurent au gré de la volonté de l'écrivain. Elles reflètent les visions du monde de leurs auteurs, et renferment en réceptacles les idéologies véhiculées par leurs sociétés. Ces villes fusionnent avec la mer et génèrent une écriture hybride aux multiples réverbérations.

Loubna Achheb, Department of French Language and Literature, Mohamed Lamine Debaghine-Sétif 2 University, 19000 Setif, lou.achheb@yahoo.fr, http://orcid.org/0000-0002-5707-1489 
Les deux auteurs algériens des deux romans étudiés, à savoir, Mohamed Dib à travers son roman Cours sur la rive sauvage et Yasmina Khadra dans son œuvre L'Olympe des infortunes, ont construit leurs fictions sur les fondements de cités sans noms, des villes dont les toponymes ont été gommés délibérément.

Ces villes et ces mers anonymes ouvrent de larges perspectives de lecture. Elles donnent lieu à une forme ludique du décodage car la curiosité nous mène, d'abord, à relever les indices de l'origine de ces villes et de ces mers délibérément rayées de la cartographie du monde par les auteurs. Ensuite, elle nous conduit sur les traces des différents mirages et métissages se trouvant au sein des œuvres analysées.

Cette étude repose, essentiellement, sur la géocritique dont Bertrand Westphal est le leader. Westphal se base sur les trois points suivants pour construire son positionnement par rapport à ce sujet : d'abord il effectue " Une réflexion sur la spatio-temporalité » (Westphal, 2007, p. 17) où il cherche à démontrer une mise en avant de l'espace par rapport au temps. Ensuite, il se focalise « [...] sur une constante de l'espace contemporain : sa mobilité [...] Y aurait-il désormais un état permanent de trangression, [...], qui ferait de tout espace un ensemble foncièrement fluide ? » (p. 17). Enfin, il met en lumière « la référentialité, la nature du lien entre le réel et la fiction, entre les espaces du monde et les espaces du texte » (p. 17).

Ainsi, nous exploitons l'espace " référentiel » dans le premier axe de notre article intitulé : De la ville anonyme à la mer ou le jeu des indices, puis l'espace « transgressif » et «mobile » dans le deuxième chapitre intitulé : Mirages et récits : le métissage au bout du chemin. Et nous utilisons l'idée de la primauté de l'espace comme le pilier sur lequel reposent les deux axes.

\section{De la ville anonyme à la mer ou le jeu des indices}

La cartographie littéraire est l'un des points nodaux sur lesquels repose la géocritique moderne. Il semble que l'espace réel soit très souvent mis en parallèle avec sa projection fictionnelle pour tenter de relever les points de convergence et de divergence qui, soit relient la réalité à la fiction, soit les séparent indéfiniment dans le but de dessiner les contours de l'idéologie de l'auteur.

Il arrive souvent $[\ldots]$ que la carte $[\ldots]$ configure sans détour l'application de la géométrie à une vision du monde intéressée et donc intéressante pour l'observateur. La carte est l'instrument d'une domestication du territoire de l'Autre, qui lui-même subit une extranéation subtile, mais inexorable (Westphal, 2011, pp. 220-221).

Mais quelle est donc l'étendue significative de l'espace sans nom au sein des deux romans étudiés ? Quel est l'objectif de ce choix délibéré par les auteurs ? Comment dépasser à travers une lecture critique le gommage de ces espaces ? 
Les deux auteurs étudiés, Mohamed Dib et Yasmina Khadra, ont érigé des villes anonymes en dualité avec la mer. Ces deux espaces : la cité et la mer, évoluent chez les deux auteurs en binarité et fusionnent pour projeter leurs visions du monde.

Il est assez intéressant de constater que les deux auteurs émiettent, tout au long de leurs romans, des indices pour permettre au lecteur de localiser l'origine des villes qu'ils annoncent comme anonymes mais qui se dévoilent au fil des mots et des fluctuations des récits.

Le premier indice, à mettre en lumière, est les prénoms des personnages des deux romans. D'abord le roman Cours sur la rive sauvage de Dib, publié en 1964, fait partie de la littérature postcoloniale. L'anonymat de la ville et de la mer relèvent, en apparence, des attributs du nouveau roman pour déstabiliser le récit et mener l'œuvre vers une véritable crise identitaire. Cependant, une analyse en profondeur nous permet de voir que Dib joue avec les mots et fait participer le lecteur à son jeu sur l'illusion.

Les trois personnages principaux de l'histoire sont les seuls à avoir un prénom mais le nom n'est pas exploité par l'écrivain car il semble plus que révélateur de l'origine des protagonistes.

Iven-Zohar est le personnage principal et narrateur de l'histoire. Il s'agit du récit de son errance, d'une ville chimérique à une autre encore plus fantasque que la précédente, à la recherche de sa bien-aimée Radia. Le troisième protagoniste est Hellé.

Pour commencer, les trois prénoms ont des origines diverses, mais toutes sont méditerranéennes. Iven est d'abord un prénom européen « forme russe du prénom grec Iohannês, devenu Jean en français » (Encyclopédie Larousse). L'auteur a choisi de mettre un « $\mathrm{e}$ » au lieu d'un « a » à ce prénom car Iven (even) en anglais renvoie à l'idée d'égalité. Zohar veut dire « chance » en arabe.

Le prénom composé "Iven-Zohar » éclaire le lecteur sur la chance du personnage d'avoir une appellation hybride parallèle à ses propres origines et à l'histoire de son pays. Ainsi, ce prénom est en relation avec la Grèce, la France, et renvoie à l'idée d'égalité. La philosophie de l'égalité peut, d'un côté, être comprise comme le système économique égalitaire russe renvoyant au communisme. Elle peut, d'un autre côté, faire penser à la philosophie existentialiste de l'absurdité d'Albert Camus qui se focalise, essentiellement, sur le désintérêt de l'être de tout ce qui l'entoure car tout lui est égal comme le révèle, si bien, Camus dans son roman L'Etranger.

Iven-Zohar est de la même origine que Radia, l'amour de sa vie. Le prénom de Radia est d'origine arabe et signifie la femme satisfaite.

Hellé, le troisième protagoniste, provient de la mythologie grecque. Cette dernière porte le prénom d'une «princesse béotienne. Fille d'Athamas, elle s'enfuit avec son frère Phrixios, promis comme elle à un sacrifice, sur un bélier 
à toison d'or. En route, elle tomba dans la mer. On donna à cette mer le nom, l'Hellespont » (Encyclopédie Larousse).

Hellespont, géographiquement, est un « canal ou détroit qui sépare l'Europe et l'Asie, et qui est indifféremment nommé par les modernes, le bras de S. Georges, les bouches de Constantinople, le détroit de Gallipoli, ou le détroit des Dardanelles » (Encyclopédie de Diderot).

Hellé est d'origine grecque et se transforme dans ce canal en Déesse. Cette mer est en même temps l'endroit qui sépare l'Europe de l'Asie et la source de jouvence du personnage. Elle lui permet de se transformer en être suprême au fond de cette étendue d'eau car l'aire de l'entre deux est le meilleur endroit pour une métamorphose.

En récapitulant l'ensemble des données rassemblées, il est évident que le pays dont nous parlons a un passé commun avec la Grèce, la France, la Turquie, une référence à d'anciens colonisateurs ce qui explique la noyade d'Hellé entre la Grèce et la Turquie. A travers cela, l'auteur met en lumière le fait que les Turcs aient noyé la colonisation européenne sur les terres du pays que nous cherchons.

Ce pays a donc des origines arabes, et a une relation avec des pays méditerranéens européens tels que : la Grèce, la France. Il a un lien avec la Turquie qui se trouve être entre l'Europe et l'Asie. Il est, également, proche de l'idéologie communiste russe donc il est loin du système capitaliste. Ce qui nous pousse à penser qu'il s'agit de l'Algérie car lors des années soixante - soixante-dix elle était socialiste et elle a vu défiler sur ses terre un bon nombre d'envahisseurs : les Grecs, les Arabes, les Turcs, les Français. Et pour renforcer notre réflexion, la théorie « égalitaire » d'Albert Camus qui est Algérien d'origine française nous conduit à positionner cette ville en Algérie. Il s'agit, évidemment d'une ville côtière. La mer en face de cette ville ne peut être que la Méditerranée.

Dans un second temps, le roman de Yasmina Khadra, publié en 2010, foisonne d'une multitude de personnages aux prénoms et noms d'origines diverses. Cela nous semble similaire à la technique utilisée par Dib.

Le premier signe à prendre en compte est la révélation de l'auteur sur le nom de la mer de son roman qui n'est autre que la Méditerranée. Maintenant, il reste à révéler sur quel continent se trouve cette ville.

Le prénom de Négus est un «titre des rois d'Ethiopie » (Dictionnaire Larousse). Négus mène Clovis à la baguette et le traite en esclave en sachant que Clovis est le prénom d'un « chef franc » (Encyclopédie Larousse) qui a pu «créer un royaume à l'échelle de toute la Gaule » (Encyclopédie Larousse). Les personnages du roman ont inversé les rôles du maître et de l'esclave qui dans la réalité sont attribués successivement à l'homme blanc qui se considère comme supérieur à l'homme noir. Cette inversion est une déformation du reflet de la ville sur le miroir de la mer pour aider l'auteur à critiquer l'idéologie esclavagiste, coloniale 
et l'esprit impérialiste importés d'Europe. Nous nous penchons, donc, à travers ces révélations du côté du continent africain.

Quelques prénoms en arabe comme : Ach (celui qui a vécu), Bliss (le diable), les frêres Zoudj (deux), Mama (maman) et Dib (nom de l'auteur introduit dans le roman) nous conduisent à croire que la ville se trouve quelque part au Maghreb et plus précisément en Algérie grâce à l'insertion du nom de l'auteur Dib dont l'origine est algérienne.

Ce qui renforce cette idée est le nom d'Ait Cétéra d'origine kabyle, une des ethnies berbères originaires d'Algérie.

Ce qui est apparent, à travers l'analyse des noms des personnages des deux romans, c'est de constater que les villes anonymes chez les deux écrivains montrent la réalité cosmopolite de l'Algérie et mettent en lumière son caractère hybride, donc les deux auteurs se focalisent sur la ville reflet d'un pays lui-même reflet du monde.

Le deuxième indice digne d'intérêt est une métaphore qui joue le rôle dans les deux romans d'un symbole emprunté d'un point de vue intertextuel à l'œuvre de Kateb Yacine intitulée Nedjma.

« Nedjma » ou «étoile » est l'emblème de la nouvelle Algérie postcoloniale qui pourtant reste fidèle à l'Algérie coloniale de Kateb Yacine car son roman Nedjma publié en 1956 était déjà selon certains critiques le symbole de l'Algérie. Kateb Yacine explique son obsession de l'Algérie éternisée par l'écriture qui devient virale chez nos deux auteurs : « [...] pour reprendre les termes $[\ldots]$ de l'écrivain [...] : 'L'Algérie n'a pas fini de venir au monde'» (Aurbakken, 1984, p. 204).

Cette métaphore de l'astre est un indice exploité par Dib dans son roman Cours sur la rive sauvage. L'auteur utilise cette image de l'étoile dans la scène du mariage d'Even-Zohar avec Radia pour aider le lecteur dans sa quête de l'origine de la ville et de la mer anonymes. Ce mariage se teinte d'une apparence funéraire car le personnage Even-Zohar se fait poignarder par sa femme Radia. Suite à cela Even-Zohar voyage de ville en ville mais reste pourtant toujours dans la même ville car ce qu'il a subi lui a donné le pouvoir de rentrer dans un monde fantastique où tout se meut en boucle.

[...] l'un de ses suivants, lui présenta une boîte faite d'un métal vert [...] Radia en retira [...] : une aiguille de lumière [...] elle me l'enfonça dans la poitrine [...] Elle me donna cinq coups consécutifs. Cinq étoiles de sang s'ouvrirent en cercle sur ma poitrine (Dib, 1964, p. 16).

Le drapeau algérien est, donc, ainsi décrit dans la scène précédente à travers ses trois couleurs : le vert correspond à la couleur de la boîte de laquelle Radia retire l'aiguille, le rouge est la couleur du sang qui jaillit de la poitrine du héros du roman sous la forme d'une étoile, le blanc est la lumière de l'aiguille qui sert à piquer Iven-Zohar. L'étoile de Dib est celle de Kateb et ne peut être que l'Algérie. 
Yasmina Khadra utilise, à son tour, l'étoile comme une métaphore renvoyant à l'Algérie ou Nedjma de Kateb Yacine. L'auteur cultive ce symbole à travers sa description de la scène de la mort d'Haroun :

\begin{abstract}
A cet instant précis, une étoile filante descend du ciel et vient s'écraser contre la mer [...] Quand elle touche la terre, elle prend une forme humaine.

-Maman, sanglote Haroun.

C'est une vieille femme accablée, tout en chagrin. Elle remonte la plage vers la dune où les quatre hommes en noir attendent. Haroun se lève au ralenti ; lui aussi se met à rayonner doucement, pareil à une flamme bleue [...] si transparent qu'on peut le traverser du regard [...] Il marche vers la femme [...] Tous les deux, la main dans la main, ils dévalent la plage, marchent sur l'eau et s'éloignent dans les ténèbres jusqu'à ce qu'ils s'éteignent, tels des lumignons soufflés par le vent (Khadra, 2010, p. 98).
\end{abstract}

L'étoile filante n'est autre que la mère d'Haroun et donc symbolise l'Algérie tant convoitée par les protagonistes et jamais retrouvée. Le seul moment qui permet au protagoniste de rejoindre, enfin, sa mère nourricière et amour éternel est celui de sa mort. Elle est, donc, ce paradis perdu, cet Olympe évanescent et impalpable. Elle joue au jeu des reflets et tente de se frayer un chemin derrière ses multiples masques qui lui octroient une véritable nature hybride.

Ce jeu binaire entre la ville et la mer, créé par les deux auteurs, permet au lecteur de se mettre en quête des indices parsemés pour, enfin, révéler la nature métisse de ce pays déguisé en ville, lui-même n'étant que l'écho du monde.

\title{
3. Mirages et récits : le métissage au bout du chemin
}

La ville en face de la mer, deux espaces tellement différents l'un de l'autre car le premier est un espace citadin et le deuxième fait partie de la nature. Les deux auteurs tentent de rassembler ces deux aires. Cette ultime fusion façonne des mirages au sein des deux romans et contribue à révéler la réalité métisse des espaces urbains et de leur rapport au monde moderne.

La mer se trouve au service de la fiction et joue le rôle du miroir reflet de la réalité de l'auteur. Pour Fanguin (2009), elle est le miroitement des premières origines de la société.

L'eau [...] marre, lac, voire mer, [...] elle [...] devient attirante ou inquiétante lorsque, de l'autre côté du miroir, elle cache un univers de mystère ou un abîme sans fond [...] en deçà du miroir de l'eau, plus on s'enfonce plus on remonte à l'origine de la vie, en une sorte de quête du paradis perdu (p. 39).

La mer Méditerranée projette les visions du monde des deux écrivains qui semblent similaires sur plusieurs points. Les villes anonymes se dotent à leur tour des attributs de la mer pour créer une écriture mirage dont le but ultime est la révélation de l'hybridité de la ville répondant aux normes de l'ère de la mondialisation. 
Les deux auteurs utilisent plusieurs techniques d'écriture pour ériger leurs villes-mers anonymes en mirage métis.

La première technique est la mise en abîme considérée comme «[...] un procédé narratif de nivelage [...] Gide mentionne comme exemple du procédé décrit par lui le livre dans le livre ou le théâtre dans le théâtre " (Meyer-Minnemann \& Schlickers, 2010, p. 93). Ce procédé est le noyau du jeu de reflets créé par les deux écrivains.

Ainsi, Dib et Khadra projettent les reflets de leurs villes sur la mer omniprésente dans les deux romans et ces villes se transforment, se métamorphosent, se décuplent puis se retrouvent selon un rythme frénétique.

Ces villes se mettent, donc, face à leurs reflets. Dib (1964) décentre la ville et la multiplie en une variété de villes similaires les unes aux autres.

Mais on sent la ville tendue et sur le point de rompre sur l'impulsion d'une puissance massive. Quelquefois elle est même foudroyée, arrêtée à l'une de ses éphémères apparences. Cette apparence heureusement ne dure jamais bien longtemps (p. 49).

Toutes ces villes reflets finissent par fusionner pour n'être plus qu'une seule et unique ville « Etoile » ou « Nedjma » pour revenir à l'image obsessionnelle réitérée sans cesse par l'auteur renvoyant à l'Algérie car la ville qui se trouve dans le pays se transforme, soudain, en pays ville du monde.

Khadra (2010) véhicule également l'image de la ville pays. Le choix de ce dernier de créer une décharge publique (appelée Olympe par les Horr ou les marginaux) est sa manière à lui de façonner le reflet de la ville. Si le terrain vague est le reflet de la cité interdite, il s'ensuit que la métropole n'est, à son tour, que le reflet du pays qu'elle représente.

[...] et par-delà l'horizon, un peu comme une hallucination, un peu comme une obsession, il verra se silhouetter les carrures sombres et inclémentes d'un pays de caillasses et de massues qui n'a rien d'une ville et rien d'un terrain vague ; un pays pire que l'enfer, pire que la folie, et Junior ne songera jamais plus à refaire sa vie (p. 188).

Mais ce pays n'est que le reflet du monde. Un reflet dénigré par les personnages de l'histoire car cette ville-pays-monde représente le côté obscur de la modernité ou plus précisément du système capitaliste qui balaye toute humanité sur son passage : « [...] un monde imbu de ses vitrines fallacieuses et de ses boulevards grouillants de gens qui s'ignorent, chacun étant aussi fermé aux autres qu'un coffre-fort dont on aurait oublié le code » (Khadra, 2010, p. 172).

Les deux auteurs utilisent des métaphores qui relient la ville et la mer. « On opère par la métaphore une recatégorisation subjective et imaginaire (on parle de recatégorisation lorsqu'un humain peut être assimilé à un animal, une réalité abstraite à un objet concret, etc.) » (Fromilhague, 2010, p. 59). Ainsi, une mise en 
abîme se crée lorsque la ville est reflétée par la mer, en même temps que la mer jette son dévolu sur la ville.

Dib (1964) insuffle la vie à la ville et lui permet de se déplacer vers la mer dans le but de pouvoir contempler son reflet : « Dévalant vers cette mer entrevue, la ville pirouettait sur un pied. C'était une ronde d'édifices, de jardins hérissés d'arbres et profilés sur la réverbération de la baie» (p. 10).

L'auteur fait se déplacer la mer dans la ville et emprunte à cette dernière le pouvoir de fluidité qu'il octroie à son personnage principal.

Et le trolley ne roula plus, le monde s'épaissit, eaux et nuées me cernèrent d'un tourbillon, éteignirent mes sens, préparant, vertigineuse, la vague qui ne se retirerait pas, qui m'emporterait. Je serrai les paupières. Je les rouvris. Il roulait toujours (Dib, 1964, p. 9).

C'est cette « vague » qui permettra à Iven-Zohar de se déplacer d'une ville à une autre et d'un monde à un autre au sein d'un univers fantasmé par l'auteur et sorti tout droit de son imagination.

Yasmina Khadra met en parallèle l'espace du terrain vague avec celui de la ville interdite de Noé qui fut, autrefois, submergée par les eaux. L'Arche de Noé se trouverait, selon le personnage Haroun, enfouie quelque part sous cette décharge à ciel ouvert. La présence de l'Arche dans l'imaginaire du protagoniste est une projection d'une future purification par les eaux de la mer du terrain vague et de la métropole. Donc, ce bateau fantôme, sorti de la nuit des temps, représente l'espoir de créer par la fusion entre la mer et la ville un monde meilleur qui transcende la fabulation en illuminant la vérité.

La deuxième technique est l'intertextualité révélée par la combinatoire du système binaire entre la ville et la mer. Genette la décrit de la manière suivante : «[...] sous le nom d'intertextualité. Je la définis [...] par une relation de coprésence entre deux ou plusieurs textes [...] par la présence effective d'un texte dans un autre » (Genette, 1982, p. 8). Elle se manifeste à travers des reprises de plusieurs histoires : l'enfer de Sisyphe, Edipe roi, les villes interdites de Sodome et de Noé. Les deux auteurs s'inspirent de la mythologie grecque, des histoires des prophètes des religions monothéistes, et des personnages des contes pour mettre en lumière une forme hybride au sein de leurs diégèses. Cette fusion entre plusieurs genres répond aux exigences de l'écriture moderne telle que considérée par Maingueneau : «On ne peut se cacher cependant que les représentations aujourd'hui dominantes de la littérature considèrent qu'une œuvre véritable doit échapper à la généricité » (Maingueneau, 2004, p. 175).

Ces histoires exploitées dans les deux textes remontent à la surface du flux des deux romans sous la forme de mirages générés par la mise en parallèle de la ville et la mer. 
Sisyphe est le premier personnage que les deux auteurs ressuscitent de la mythologie grecque. Et chacun l'utilise pour projeter l'enfer de l'éternel recommencement dans lequel baigne l'humanité.

Dib (1964) hybride sa fiction et insère dans sa chair des bribes d'histoires du monde. Il fait subir, à son personnage principal Iven-Zohar le même sort que celui de Sisyphe.

Je veux jeter un regard implorant vers la mer; je l'entrevois à une effrayante profondeur qui cogne des rochers et mugit [...] Je lève les yeux [...] sur l'étrangère [,..], elle se révèle si horrible [...] Une voix me souffle qu'elle m'a hissé à cette altitude pour m'en précipiter ensuite [...] je m'abîme dans le gouffre bouillonnant. Sa main avec, au milieu l'étoile rouge, est restée levée devant mes yeux. Je ne sais combien d'heures je suis tombé de la sorte, combien de fois je suis mort, combien de fois j'ai repris vie, avant de me retrouver au même endroit gisant sur la plage. La mer jouait avec mes cheveux et le soleil flamboyait au zénith (pp. 86-87).

Iven-Zohar découvre son supplice de la boucle de "l'abîme » infini après avoir contemplé la mer qui lui permet de voir Hellé telle quelle, sans aucun artifice, à l'image de l'éternelle ville-pays car sa main est marquée de l'étoile rouge symbole de l'Algérie et emblème central du drapeau algérien. Hellé, déesse grecque, fusionne avec l'étoile algérienne pour donner au lecteur un aperçu sur les origines métisses de l'Algérie sous la forme d'un mirage évanescent.

Khadra (2010) utilise son personnage Haroun (un marginal du terrain vague) comme la réincarnation de Sisyphe. Pour Khadra : "Haroun est Sisyphe valétudinaire » (p. 25) car tous les jours il creuse des trous pour déterrer l'Arche de Noé sous le tronc d'un arbre, le soir la montée des eaux comble les trous de sable qu'il recreuse à l'infini. L'histoire de Sisyphe est générée et perpétuée par la mer car les trous creusés par Haroun pour déterrer le bateau sont effacés par les vagues.

La mer crée l'enfer de l'éternel recommencement et vise à faire éclore cette histoire comme mirage reflet du terrain vague qui remet en scène toutes les injustices de la ville interdite. Ces injustices découlent du règne animal car le plus fort s'en prend au plus faible comme c'est le cas sur ce faux Olympe car le Pacha, personnage le plus fort des marginaux, règne en maitre absolu. Cette critique de la ville moderne émerge de la création du mirage suite à la fusion entre la ville et la mer.

Les deux écrivains hybrident leurs textes en usant de diverses histoires. D'abord, Dib (2010) donne à la mer le pouvoir de la Gorgone (mythologie grecque) car elle change Iven-Zohar en statue. Il reprend, également, des éléments spécifiques au conte tels que les dragons, la forêt qu'il replace dans son texte :

Nous partîmes,

Dragons remuant le fond de l'avenue, des eaux et des nuées envahies de mouettes attaquaient, sans l'atteindre, l'or du ciel [...] mais dévoraient les hauteurs, les villas [...] Nous nous réfugiâmes dans une forêt où les chemins s'entrecroisaient sans trouver d'issue, puis nous revînmes vers la perspective balayée par la charge des vagues. Nous débouchâmes sur un monde de flammes (p. 7). 
La mer, ses eaux, en combinaison avec la ville projettent les éléments du conte dans le roman dibien, puis les font échouer dans un vaste labyrinthe (grec).

Ce labyrinthe est celui de l'écriture dibienne qui tourne en rond en usant de la combinatoire binaire ville/mer pour créer des mirages dans le but de révéler le métissage de l'écriture et celui de la société.

Ensuite, Khadra (2010) s'inspire des histoires des prophètes : Noé et Loth pour créer ses mirages. Celle de Noé introduit l'enfer de Sisyphe comme nous l'avons vu précédemment. Et celle de Loth, justifie le rejet du protagoniste Ach de la ville moderne car elle se transforme en reflet de la ville interdite de Loth.

Surtout, ne te retourne pas, lui recommande Ach en agitant le doigt [...] Loth a rassemblé sa tribu et lui a expressément intimé de pas se retourner quand elle entendra le bon Dieu foutre en l'air Sodome. Mais la femme de Loth, elle, elle s'est retournée [...] (pp. 13-14).

L'auteur met en parallèle les deux villes de Loth et celle d'Ach de la façon suivante :

[...] J'ai pensé $[\ldots]$ pouvoir faire le malin et regarder une dernière fois la ville dans le rétroviseur. Mais le bon Dieu. Il se contente pas de donner des ordres, il veille au grain aussi : paf !le rétroviseur me pète à la figure. C'est comme ça que j'ai perdu mon œil (p. 14).

Le point commun entre les deux villes est la malédiction car Ach, qui se retourne pour voir sa ville une dernière fois avant son départ pour son Olympe (décharge publique), perd son œil suite à l'explosion du rétroviseur. Ce châtiment est similaire à celui d'Edipe roi qui se crève les yeux pour ne plus subir la fiction de la vie. Ach n'a plus qu'un œil, ce qui lui permet de basculer, facilement, de la fiction à la réalité au sein du récit khadrien.

Khadra mélange entre le conte et le roman en s'inspirant de l'histoire de Blanche-Neige lorsque le Pacha s'adresse à Ben Adem : « - Tu restes où t'es, Blanche-Neige, crie pompeusement le Pacha pour couvrir son recul, sinon, je te marche dessus jusqu'à te faire sortir tes tripes par les oreilles [...] » (Khadra, 2010, p. 120).

Cette appellation est due à l'apparence blanchâtre de Ben Adem (fils d'Adam) qui apparaît soudain sur le terrain vague et fait des discours prophétiques sur l'humanité, ses heures de gloire et d'horreur. Mais la relation entre Ben Adem et Blanche-Neige n'est pas qu'une question de couleur, elle est plutôt en rapport avec le miroir magique de la belle-mère qui ne peut refléter que la vérité. La mer serait donc la réincarnation de ce miroir car elle reflète les courbes de la ville sur le terrain vague. Elle y reflète parallèlement la réalité métisse du monde moderne. 


\section{Conclusion}

Les deux romans étudiés de Mohamed Dib et Yasmina Khadra s'entremêlent et rejoignent la même vision. Celle de représenter, à travers le prisme de l'espace, le monde moderne capitaliste sous sa véritable apparence.

Ces deux écrivains ont opté pour la mise en scène de villes anonymes face à la mer. Ces deux espaces évoluent dans les deux textes à travers un système binaire dynamique et créateur de mirages, de jeux de reflets pour faire miroiter la réalité du métissage de leurs écritures.

Ils ont, donc, réussi en créant l'anonymat de leurs villes à les cartographier à travers un jeu d'indices impliquant le lecteur et le conduisant à résoudre l'énigme de l'identité des cités et des mers de ces récits.

Ils ont reposé leurs stratégies d'écriture sur les deux modes de la mise en abîme et de l'intertextualité pour mettre en lumière le rapport ombilical liant la ville à la mer. De la fusion entre ces deux espaces naissent des mirages révélateurs de l'hybridité de leurs romans, de la société algérienne et du monde.

\section{References}

Aurbakken, K. (1984). L'étoile d'araignée. Une lecture de Nedjma de Kateb Yacine. Paris: Publisud. Dib, M. (1964). Cours sur la rive sauvage. Paris: Seuil.

Dictionnaire Larousse. Retrieved March 20, 2020, from https//www.larousse.fr/.

Encyclopédie de Diderot. Retrieved March 20, 2020, from https//encyclopdie.eu/.

Encyclopédie Larousse. Retrieved March 20, 2020, from https://www.larousse.fr/encyclopedie/.

Fanguin, D. (2009). Le psychisme. Paris: Ellipses.

Fromilhague, C. (2010). Les figures de style. Paris: Armand Colin.

Genette, G. (1982). Palimpseste. Paris: Seuil.

Khadra, Y. (2010). L'Olympe des infortunes. Paris: Julliard.

Maingueneau, D. (2004). Le discours littéraire. Paratopie et scène d'énonciation. Paris: Armand Colin.

Meyer-Minnemann, K., \& Schlickers, S. (2010). La mise en abyme en narratologie. In J. Pier, \& F. Berthelot (Ed.), Narratologies contemporaines. Approches nouvelles pour la théorie et l'analyse du récit (pp. 91-108). Paris: Editions des archives contemporaines.

Westphal, B. (2007). La géocritique. Réel, fiction, espace. Paris: Minuit.

Westphal, B. (2011). Le monde plausible. Espace, lieu, carte. Lonrai: Minuit. 